\title{
Raus aus der Klinik, rein in die Klinik: Wer ist gefährdet?
}

- Viele Patienten müssen, gerade aus dem Krankenhaus entlassen, ungeplant wieder aufgenommen werden. Wer besonders gefährdet ist, lässt sich anhand von sieben Faktoren vorhersagen.

Dr. Jacques Donzé et al., Brigham and Women's Hospital in Boston, haben einen Score entwickelt, mit dem sich Patienten mit hohem Risiko für eine vermeidbare Wiederaufnahme identifizieren lassen. Der Score mit dem Namen HOSPITAL beinhaltet sieben unabhängige Risikofaktoren für Wiedereinweisungen innerhalb von 30 Tagen, die weder planmäßig noch aufgrund einer neu aufgetretenen Erkrankung erfolgen. HOSPITAL steht für:

- Hb-Wert bei Entlassung $<12$ g/dl

(1 Punkt),

- Entlassung aus einer onkologischen Abteilung (2 Punkte),
- Na-Spiegel (englisch: Sodium) bei Entlassung $<135 \mathrm{mmol} / \mathrm{l}$ (1 Punkt),

- Prozeduren während des Aufenthalts (z. B. Biopsie, Transfusion, Endoskopie) (1 Punkt), - Index-Aufnahme-Typ: nicht elektiv (1 Punkt),

- Aufnahmen im vorausgegangenen Jahr (0: 0 Punkte, 1-5: 2 Punkte, $>$ 5: 5 Punkte),

- Länge des Aufenthalts $\geq 5$ Tage (2 Punkte).

Nach der Gesamtpunktzahl eines Patienten wird sein Risiko für eine vermeidbare Wiedereinweisung als niedrig (0-4), mittel (5-6) oder hoch ( $\geq 7$ Punkte) eingestuft. Zur Entwicklung und Validierung des Scores wurden Daten von 10731 Entlassungen aus Abteilungen der Inneren Medizin des Brigham and Women's Hospital ausgewertet. 2398 Patienten (22,3\%) waren innerhalb von 30 Tagen erneut in der Klinik gelandet. Bei 879 wurde die Wiederaufnahme als vermeidbar angesehen. Anhand der Daten von 6141 Patienten der Gesamtstichprobe wurden in einer multivariaten Regressionsanalyse die genannten Risikofaktoren für eine Wiederaufnahme herausgefiltert. Gemäß des HOSPITAL-Scores wurde knapp der Hälfte dieser Patienten ein niedriges Risiko zugesprochen und damit eine Wiederaufnahmequote von 5,2\% erwartet - was gut mit der tatsächlichen Rate von $5,4 \%$ übereinstimmte. Jeweils ein Viertel der Patienten hatte ein mittleres oder hohes Risiko; auch hier waren geschätzte und beobachtete Wiedereinweisungsraten weitgehend identisch, mit $9,8 \%$ vs. $9,0 \%$ und $18,3 \%$ vs. $18,7 \%$.

Der HOSPITAL-Score versetzt Ärzte in die Lage, prospektiv die Patienten auszumachen, die ein hohes Risiko für eine vermeidbare Wiederaufnahme haben, und bei diesen gezielt zu intervenieren, so die Autoren.

BS

- Donzé J et al. JAMA Intern Med 2013, online 25. März

\section{Welcher Tarif für welchen Patienten?}

Die verständliche Freude über die Abschaffung der lästigen Praxisgebühr ist rasch verklungen. Schon melden sich erste Stimmen aus der Ärzteschaft und fordern neue Instrumentarien, um die Zahl der Arztbesuche zu verringern. Der Patient müsse, so unsere Ärztefunktionäre, gesteuert oder zumindest gelenkt werden. Dazu brauche man Wahltarife.

In diesem Zusammenhang soll die KBV bereits Modelle erarbeitet haben, wobei jeder Versicherte das für ihn richtige Maß an hausärztlicher („Der weiß nichts von allem“) und fachärztlicher („Der weiß alles von nichts") Versorgung wählen kann:

\section{Nur Hausarzt, kein Facharzt:}

Dieser Tarif bietet sich für alle an, die uneingeschränktes Vertrauen zu ihrem Hausarzt haben und befürchten, dass eine fachärztliche Rundumversorgung nur dazu führe, dass man kränker werde als man eigentlich sei; gesund bedeute dann nur, unzureichend untersucht zu sein.

\section{Nur Facharzt, kein Hausarzt:}

Dieser Tarif ist der richtige für jene, die den Hausarzt für einen Universaldilettanten halten. Sie möchten ungehindert durch alle Facharztpraxen flottieren, der Arztbesuch soll zu einem Full-Time-Job werden. Folge dieses "doctor hoppings" könnte sein, dass die so Versicherten später einmal gesund sterben müssen.

\section{Facharzt nur auf Überweisung des Hausarztes:}

In diesem System wird der Hausarzt zum unverzichtbaren Lotsen, der das Schiff "Patient" sicher steuert, damit es nicht an den Klippen der Facharztpraxen zerschellt und diese nur in Ausnahmesituationen gezielt ansteuert. Somit bräuchte man auch weniger Fachärzte und könnte die überflüssigen Spezialisten zu Haus- ärzten umschulen, was aber gar nicht so einfach sein dürfte. Ein entsprechendes Curriculum gibt es nämlich noch nicht.

\section{Weder Hausarzt noch Facharzt:}

Dieser Tarif ist sicherlich sehr interessant hinsichtlich seiner Auswirkungen auf die Lebenserwartung. Ob er allerdings gut oder schlecht für die Prognose quoad vitam ist, weiß niemand so genau. Er wäre aber eine glaubwürdige Option für diejenigen, die sich für ein sozialverträgliches Frühableben stark machen, weil sie befürchten, ansonsten sei das Gesundheitssystem, aber auch das Rentensystem gar nicht mehr zu retten.

Bevor ein Versicherter sich nun für einen dieser Tarife entscheidet, wird er sich sicherlich von Ihnen beraten lassen und dabei vielleicht auch die Frage stellen: Welchen Tarif bevorzugen eigentlich Sie, Herr Doktor? Dr. Peter Stiefelhagen . 